\title{
CONVERGENCE OF A NORMALIZED GRADIENT ALGORITHM FOR COMPUTING GROUND STATES
}

\author{
ERWAN FAOU AND TIPHAINE JÉZÉQUEL
}

\begin{abstract}
We consider the approximation of the ground state of the one-dimensional cubic nonlinear Schrödinger equation by a normalized gradient algorithm combined with linearly implicit time integrator, and finite difference space approximation. We show that this method, also called imaginary time evolution method in the physics literature, is convergent, and we provide error estimates: the algorithm converges exponentially towards a modified solitons that is a space discretization of the exact soliton, with error estimates depending on the discretization parameters.
\end{abstract}

\section{INTRODUCTION}

The goal of this paper is to give a convergence proof of a normalized gradient algorithm used to compute numerically ground states of Schrödinger equations fulfilling symmetry and coercivity conditions as considered in the seminal works of Weinstein [13] and Grillakis, Shatah and Strauss [10, 11]. This algorithm is also called imaginary time method in the physics literature: see for instance $[8,1,2,7,5]$ and the reference therein. Let us describe the algorithm in the case of the focusing cubic non linear Schrödinger equation

$$
i \partial_{t} \psi=-\frac{1}{2} \Delta \psi-|\psi|^{2} \psi
$$

set on $\mathbb{R}$, where $\psi(t, x)$ depends on space variables $x \in \mathbb{R}$. With this equation is associated the energy

$$
H(\psi, \bar{\psi})=\frac{1}{4} \int_{\mathbb{R}}|\nabla \psi|^{2}-|\psi|^{4},
$$

that is preserved by the flow of (NLS) for all times. The equation (NLS) can be written

$$
i \partial_{t} \psi=-\frac{1}{2} \Delta \psi-|\psi|^{2} \psi=2 \frac{\partial H}{\partial \bar{\psi}}(\psi, \bar{\psi}) .
$$

In the rest of this paper, the notation $\nabla H$ will denote the $L^{2}$ derivative of the energy $H$ with respect to real functions $\psi$. Note that we have for a real function $u$

$$
\nabla H(u)=2 \frac{\partial H}{\partial \bar{\psi}}(u, u)=-\frac{1}{2} \Delta u-u^{3},
$$

1991 Mathematics Subject Classification. 35Q1, 35Q55, 65N12, 65J15.

Key words and phrases. Nonlinear Schrödinger equation, ground state, imaginary time method.

This work has been supported by the ERC Starting Grant GEOPARDI No 279389 . 
the left-hand side denoting the Fréchet derivative of $H(u)$ considered as a functional acting on real functions. Note that naturally, $\nabla H \in H^{-1}$ with the embedding $H^{-1} \subset L^{2} \subset H^{1}$.

With these notations, the ground state $\eta(x)$ is defined as the unique real symmetric minimizer in $H^{1}$ of the problem

$$
\min _{\|\psi\|_{L^{2}}=1} H(\psi) .
$$

In the one dimensional cubic case considered in this paper, explicit computations show that

$$
\eta(x):=\frac{1}{2} \operatorname{sech}\left(\frac{x}{2}\right) .
$$

We denote by $\lambda$ the Lagrange multiplier associated with this minimization problem, such that

$$
\nabla H(\eta)=-\frac{1}{2} \Delta \eta-\eta^{3}=-\lambda \eta .
$$

With the ground state $\eta$ is associated the solution $\eta(t, x)=\eta(x) e^{i \lambda t}$ of the time dependent equation (NLS). By using translation and scaling, the ground state gives rise to a family of explicit solutions of (NLS) that have the property to be orbitally stabe in $H^{1}$, see $[13,10,11,6]$. For instance, any solution starting close to $\eta(x)$ will remain close to the manifold $\left\{e^{i \alpha} \eta(x-\right.$ c) $\mid \alpha, c \in \mathbb{R}\} \subset H^{1}$, the rotation and translation being natural invariant group actions of the nonlinear Schrödinger equation (NLS).

In more general situations, $\eta$ is not explicitly known, and one has to rely on numerical simulations to compute it. To this aim, the imaginary time method, which is a nonlinear version of the normalized gradient algorithm is widely used. The goal of the present paper is to analyse the efficiency of this method in the simple case described above (the results obtained are in fact valid in more general situations - essentially all situations where the Grillakis-Shatah-Strauss arguments apply).

The time-discretized algorithm consists in defining a sequence a functions $\left\{\psi_{n}\right\}_{n \in \mathbb{N}}$ as follows:

(i) An intermediate function $\psi_{n}^{*}$ is defined as a numerical approximation of the solution of the parabolic equation

$$
\partial_{t} \psi=\frac{1}{2} \Delta \psi+|\psi|^{2} \psi=-\nabla H(\psi)
$$

over a time interval $[0, \tau]$, where $\tau$ is a given time step. To compute $\psi_{n}^{*}$, we will see that the best results are given by the linearly implicit method

$$
\psi_{n}^{*}=\psi_{n}-\tau \widehat{\nabla H}\left(\psi_{n}, \psi_{n}^{*}\right),
$$

where

$$
-\widehat{\nabla H}\left(\psi_{n}, \psi_{n}^{*}\right)=\frac{1}{2} \Delta \psi_{n}^{*}+\left|\psi_{n}\right|^{2} \psi_{n}^{*} ;
$$

(ii) Then we define the normalized function

$$
\psi_{n+1}=\frac{\psi_{n}^{*}}{\left\|\psi_{n}^{*}\right\|_{L^{2}}} .
$$


Note that the algorithm presented above preserves the real nature of the function $\psi$ : if $\psi_{0}$ is real valued, then for all $n \in \mathbb{N}, \psi_{n}$ is real, and that the same holds true for symmetric functions satisfying $\psi_{n}(-x)=\psi_{n}(x)$.

Our results can be summarized as follows:

- In the semi-discrete case described above, if the initial data $\psi_{0}$ is real, symmetric and sufficiently close to $\eta$, then $\psi_{n}$ converges exponentially towards $\eta$ in $H^{1}$.

- In the fully discrete case, where the discretization is space is made by finite difference in space with mesh $h$ combined with a Dirichlet cut-off for large values of $x$ (namely $x \leq K h$ ), then we prove the exponential convergence towards a modified soliton $\eta_{h, K}$ that is $\mathcal{O}\left(h+\frac{1}{h^{2}} e^{-C_{1} K h}\right)$ close to the exact soliton $\eta$.

The main property explaining the excellent performance of this method is the fact that the linearly implicit numerical scheme exactly preserves the ground state: if $\psi_{n}=\eta$, then $\psi_{n+1}=\eta$, and the same holds true for discrete in space ground states satisfying a discrete version of (1.3). This fact is very general: it holds for any linearly implicit scheme applied to a semilinear PDEs. This important feature makes of course this scheme much more attractive than other possible schemes where the nonlinearity would be approached by

$$
-\widehat{\nabla H}\left(\psi_{n}, \psi_{n}^{*}\right)=\frac{1}{2} \Delta \psi_{n}^{*}+ \begin{cases}\left|\psi_{n}\right|^{2} \psi_{n} & \text { (semi-explicit), } \\ \left|\psi_{n}^{*}\right|^{2} \psi_{n}^{*} & \text { (fully-implicit), }\end{cases}
$$

despite the fact that fully-implicit schemes enjoy the energy diminishing property for standard gradient system (see [12]). As we will discuss later, these schemes still converge, but towards modified ground states $\eta_{\tau}=\eta+$ $\mathcal{O}(\tau)$. As these results would be weaker for longer detailed proofs, we only give the main arguments to obtain them, keeping a full detailed convergence proof for the linearly implicit scheme.

\section{The Hamiltonian near the ground state}

We work in the space $V$ of real symmetric functions of $H^{1}$ :

$$
V:=\left\{\varphi \in H^{1}(\mathbb{R}, \mathbb{R}) \mid \varphi(-x)=\varphi(x)\right\} .
$$

We consider the usual $L^{2}$ and $H^{1}$ norms, and denote by $\langle\cdot, \cdot\rangle$ the canonical real scalar product on $L^{2}$ :

$$
\langle\varphi, \psi\rangle=\int_{\mathbb{R}} \varphi(x) \psi(x) \mathrm{d} s, \quad\|\varphi\|_{L^{2}}^{2}=\langle\varphi, \varphi\rangle, \quad\|\varphi\|_{H^{1}}^{2}=\|\varphi\|_{L^{2}}^{2}+\left\|\partial_{x} \varphi\right\|_{L^{2}}^{2} .
$$

2.1. Coordinates in the neighborhood of the ground state. We introduce the set of the functions $R$-close to $\eta$

$$
\mathcal{U}(R):=\left\{\varphi \in V \mid\|\varphi-\eta\|_{H^{1}}<R\right\},
$$

the set

$$
W:=\{u \in V \mid\langle u, \eta\rangle=0\}
$$

and the map $\chi$

$$
\begin{aligned}
\chi: \mathbb{R} \times W & \rightarrow V \\
(r, u) & \mapsto(1+r) \eta+u .
\end{aligned}
$$


The map $\chi$ allows to use $(r, u)$ as coordinates in $\mathcal{U}(R)$. Observe that $\chi$ is smooth with bounded derivatives and so is the inverse $\chi^{-1}$, from the explicit formula

$$
\begin{aligned}
\chi^{-1}: V & \rightarrow \mathbb{R} \times W \\
\psi & \mapsto(r(\psi), u(\psi))=(\langle\psi, \eta\rangle-1, \psi-\langle\psi, \eta\rangle \eta) .
\end{aligned}
$$

We will also use the following notations: We define the $L^{2}$ projectors

$$
P_{\eta} u=\langle u, \eta\rangle \eta \quad \text { and } \quad P_{W} u=I-P_{\eta},
$$

and we define the function

$$
\widetilde{\mathcal{H}}(r, u)=(H \circ \chi)(r, u) .
$$

We can verify the following relations:

$$
\partial_{r} \widetilde{\mathcal{H}}(r, u)=\langle\nabla H(\chi(r, u)), \eta\rangle=\eta^{-1}\left(P_{\eta} \nabla H\right)(\chi(r, u)) \in \mathbb{R},
$$

and

$$
\nabla_{u} \widetilde{\mathcal{H}}(r, u)=P_{W} \nabla H(\chi(r, u)) \in H^{-1} .
$$

Before collecting some expressions of the Hamiltonian and the gradient flow in coordinates $(r, u)$, let us introduce the following notations:

Definition 2.1. Let $p \geq 1$. We say that $R(u)=\mathcal{O}\left(\|u\|_{H^{1}}^{p}\right)$ if for all $B$, there exists a constant $C$ such that for all $u \in H^{1},\|u\|_{H^{1}} \leq B$, we have

$$
\|R(u)\|_{H^{1}} \leq C\|u\|_{H^{1}}^{p} .
$$

We will also use the notation $R(u, v)=\mathcal{O}\left(\|u, v\|_{H^{1}}^{p}\right)$ if for all $B$, there exists $C$ such that for all $u, v \in H^{1}$ satisfying $\|u\|_{H^{1}} \leq B$ and $\|v\|_{H^{1}} \leq B$, then we have

$$
\|R(u, v)\|_{H^{1}} \leq C\left(\|u\|_{H^{1}}^{p}+\|v\|_{H^{1}}^{p}\right) .
$$

Finally, a function $u=\mathcal{O}(r)$ if $\|u\|_{H_{1}} \leq C r$ for $r$ small enough and a constant $C$ independent of $u$.

With these notations and the fact that $\psi=(1+r) \eta+u$, we compute using (1.3) that

$$
\begin{aligned}
-\nabla H(\psi)= & \frac{1}{2} \Delta \psi+\psi^{3} \\
= & (1+r)\left(-\eta^{3}+\lambda \eta\right)+\frac{1}{2} \Delta u+u^{3} \\
& +(1+r)^{3} \eta^{3}+3(1+r)^{2} \eta^{2} u+3(1+r) \eta u^{2} \\
= & \lambda \eta+\frac{1}{2} \Delta u+3 \eta^{2} u+\mathcal{O}\left(r+\|u\|_{H^{1}}^{2}\right) .
\end{aligned}
$$

Note that to obtain the bound, we have used the fact that $\eta \in H^{1}$, as well as the estimate

$$
\|u v\|_{H^{1}} \leq C\|u\|_{H^{1}}\|v\|_{H^{1}},
$$

for two functions $u$ and $v$. We deduce using (2.2) and (2.3) that

$$
\begin{aligned}
\partial_{r} \widetilde{\mathcal{H}}(r, u) & =-\lambda-\frac{1}{2}\langle u, \Delta \eta\rangle-3\left\langle\eta^{3}, u\right\rangle+\mathcal{O}\left(r+\|u\|_{H^{1}}^{2}\right) \\
& =-\lambda-2\left\langle\eta^{3}, u\right\rangle+\mathcal{O}\left(r+\|u\|_{H^{1}}^{2}\right),
\end{aligned}
$$


as $\langle u, \eta\rangle=0$ and $\langle\eta, \eta\rangle=1$, and

$$
\begin{aligned}
\nabla_{u} \widetilde{\mathcal{H}}(r, u) & =-P_{W}\left(\lambda \eta+\frac{1}{2} \Delta u+3 \eta^{2} u\right)+\mathcal{O}\left(r+\|u\|_{H^{1}}^{2}\right) \\
& =-\frac{1}{2} \Delta u-3 \eta^{2} u+2\left\langle\eta^{3}, u\right\rangle \eta+\mathcal{O}\left(r+\|u\|_{H^{1}}^{2}\right) .
\end{aligned}
$$

2.2. Projection onto the unit $L^{2}$ sphere. Let us define now the function $u \mapsto r(u)$ from $W$ to $\mathbb{R}$ by the implicit relation

$$
\|\chi(r(u), u)\|_{L^{2}}^{2}=1 \text {. }
$$

By explicit computation, we get

$$
r(u)=-1+\sqrt{1-\|u\|_{L^{2}}^{2}}
$$

from which we deduce that $r(u)$ is well defined and smooth in a neighborhood of 0 in $H^{1}$, and that moreover $|r(u)|=\mathcal{O}\left(\|u\|_{L^{2}}^{2}\right)$ when $u$ is small enough. Hence, $u \mapsto \chi(r(u), u)$ is a local parametrization of $\mathcal{S} \cap V$ in a neighborhood of $\eta$, where

$$
\mathcal{S}:=\left\{\psi \in V \mid\|\psi\|_{L^{2}}=1\right\} .
$$

Note that in this parametrization, $u=0$ corresponds to the ground state $\eta$. We then define

$$
\mathcal{H}(u):=\widetilde{\mathcal{H}}(r(u), u)=H(\chi(r(u), u)) .
$$

The main result in [13], see also [10, 11, 9], is the following:

Proposition 2.2. The point $u=0$ is a non degenerate minimum of $\mathcal{H}$ : there exist some positive constants $c_{0}$ and $\rho_{0}$ such that

$$
\forall v \in W \quad d^{2} \mathcal{H}(0) .(v, v) \geq c_{0}\|v\|_{H^{1}}^{2} .
$$

Note that we have

$$
\nabla_{u} r(u)=-\frac{u}{\sqrt{1-\|u\|_{L^{2}}^{2}}}=-u+\mathcal{O}\left(\|u\|_{H^{1}}^{3}\right) .
$$

Hence, as $r(u)=\mathcal{O}\left(\|u\|_{H^{1}}\right)$, we have

$$
\begin{aligned}
\nabla_{u} \mathcal{H}(u) & =\partial_{r} \widetilde{\mathcal{H}}(r(u), u) \nabla_{u} r(u)+\left(\nabla_{u} \widetilde{\mathcal{H}}\right)(r(u), u) \\
& =\lambda u-\frac{1}{2} \Delta u-3 \eta^{2} u+2\left\langle\eta^{3}, u\right\rangle \eta+\mathcal{O}\left(\|u\|_{H^{1}}^{2}\right) \\
& =P_{W}\left(\lambda u-\frac{1}{2} \Delta u-3 \eta^{2} u\right)+\mathcal{O}\left(\|u\|_{H^{1}}^{2}\right) .
\end{aligned}
$$

From the previous proposition, we deduce the following:

Corollary 2.3. The operator $A: W \rightarrow W$ defined by

$$
A u:=P_{W}\left(\lambda u-\frac{1}{2} \Delta u-3 \eta^{2} u\right)
$$

is $L^{2}$ symmetric and positive definite in $H^{1}$ : We have

$$
c\|u\|_{H^{1}}^{2} \leq\langle u, A u\rangle \leq C\|u\|_{H^{1}}^{2}
$$

for some constants $c$ and $C$, and

$$
\nabla_{u} \mathcal{H}(u)=A u+\mathcal{O}\left(\|u\|_{H^{1}}^{2}\right) .
$$


Let us remark that the coercitivity relation (2.9) combined with CauchySchwartz inequality implies that

$$
c\|u\|_{H^{1}}^{2} \leq\langle u, A u\rangle \leq C\|u\|_{L^{2}}\|A u\|_{L^{2}} \leq C\|u\|_{H^{1}}\|A u\|_{L^{2}}
$$

for some constant $C$. We thus infer the existence of a constant $c>0$ such that

$$
\|A u\|_{L^{2}} \geq c\|u\|_{H^{1}} \quad \text { and } \quad\|A u\|_{L^{2}}^{2} \geq c\langle u, A u\rangle .
$$

\section{Continuous normalized Gradient Flow}

We consider the continuous normalized gradient flow (see for instance [4])

$$
\partial_{t} \psi=-\nabla H(\psi)+\left\langle\nabla H(\psi), \frac{\psi}{\|\psi\|_{L^{2}}}\right\rangle \frac{\psi}{\|\psi\|_{L^{2}}},
$$

which is the projection of the standard gradient flow $\partial_{t} \psi=-\nabla H(\psi)$ onto the unit $L^{2}$ sphere. The local existence of an $H^{1}$ solution to (3.1) is guaranteed by standard argument, using the fact that $\Delta$ defines a semi group in $H^{1}$, and that $H^{1}$ is an algebra in dimension 1 (see (2.4)).

3.1. The gradient flow in local variables. We assume for the moment that $\psi(t)$ remains in a ball sufficiently close to $\eta$ so that we can write $\psi(t)=$ $(1+r(t)) \eta+u(t)$ with $r(t)>-1$ and $u(t) \in W$. We have in this case $P_{\eta} \psi=(1+r) \eta$ and $P_{W} \psi=u$. Note also that

$$
\begin{aligned}
\langle\nabla H(\psi), \psi\rangle & =\left\langle P_{\eta} \nabla H, P_{\eta} \psi\right\rangle+\left\langle P_{W} \nabla H, P_{W} \psi\right\rangle \\
& =(1+r) \partial_{r} \widetilde{\mathcal{H}}+\left\langle\nabla_{u} \widetilde{\mathcal{H}}, u\right\rangle
\end{aligned}
$$

and that

$$
\|\psi\|_{L^{2}}^{2}=(1+r)^{2}+\|u\|_{L^{2}}^{2} .
$$

It turns out that the relation $\|\psi\|_{L^{2}}^{2}=1$ implies that $\|u\|_{L^{2}} \leq 1$. In the following we will only work with functions $u$ satisfying this condition. Applying successively the operators $P_{\eta}$ and $P_{W}$ to the equation (3.1), we obtain the relation

$$
\begin{aligned}
\partial_{t} r & =-\partial_{r} \tilde{\mathcal{H}}(r, u)+\frac{(1+r)^{2}}{(1+r)^{2}+\|u\|_{L^{2}}^{2}} \partial_{r} \widetilde{\mathcal{H}}+\frac{(1+r)}{(1+r)^{2}+\|u\|_{L^{2}}^{2}}\left\langle\nabla_{u} \widetilde{\mathcal{H}}, u\right\rangle, \\
\partial_{t} u & =-\nabla_{u} \widetilde{\mathcal{H}}(r, u)+\frac{(1+r) u}{(1+r)^{2}+\|u\|_{L^{2}}^{2}} \partial_{r} \widetilde{\mathcal{H}}+\frac{u}{(1+r)^{2}+\|u\|_{L^{2}}^{2}}\left\langle\nabla_{u} \widetilde{\mathcal{H}}, u\right\rangle .
\end{aligned}
$$

As the $L^{2}$ norm of $\psi$ is preserved, we calculate directely that $(1+r)^{2}+$ $\|u\|_{L^{2}}^{2}$ is preserved along the flow of this system (and is constant equals to one), and that we can solve $r$ in terms of $u$ as above (see (2.5)). We thus obtain the closed equation

$$
\partial_{t} u=-\nabla_{u} \widetilde{\mathcal{H}}(r(u), u)+(1+r(u)) u \partial_{r} \widetilde{\mathcal{H}}(r(u), u)+u\left\langle\nabla_{u} \widetilde{\mathcal{H}}, u\right\rangle .
$$

But we have

$$
\left(\nabla_{u} \widetilde{\mathcal{H}}\right)(r(u), u)=\nabla_{u} \mathcal{H}(u)+\partial_{r} \widetilde{\mathcal{H}}(r(u), u) \frac{u}{\sqrt{1-\|u\|_{L^{2}}^{2}}},
$$


hence we can write the equation (3.2) as

$$
\begin{aligned}
\partial_{t} u= & -\nabla_{u} \mathcal{H}(u)+u\left\langle u, \nabla_{u} \mathcal{H}(u)\right\rangle-\partial_{r} \widetilde{\mathcal{H}}(r(u), u) \frac{u}{\sqrt{1-\|u\|_{L^{2}}^{2}}} \\
& +u \sqrt{1-\|u\|_{L^{2}}^{2}} \partial_{r} \widetilde{\mathcal{H}}(r(u), u)+\partial_{r} \widetilde{\mathcal{H}}(r(u), u) \frac{u\|u\|_{L^{2}}^{2}}{\sqrt{1-\|u\|_{L^{2}}^{2}}} .
\end{aligned}
$$

and hence

$$
\partial_{t} u=-\nabla_{u} \mathcal{H}(u)+u\left\langle u, \nabla_{u} \mathcal{H}(u)\right\rangle,
$$

which is the gradient flow in local coordinates $(r(u), u)$ on $\mathcal{S}$. Note that with the notation of the previous paragraph, we we can write this equation as follows:

$$
\partial_{t} u=-A u+R(u)
$$

with $R(u)=\mathcal{O}\left(\|u\|_{H^{1}}^{2}\right)$.

3.2. Convergence of the flow. We prove now that the solution of the normalized gradient flow (3.1) converges towards the ground state $\eta$ if the initial value sufficiently close to it.

Theorem 3.1. There exists $\mu>0$ such that if $u(t) \in W$ is a solution of (3.3) with $\left\|u_{0}\right\|_{H^{1}}$ sufficiently small, then we have

$$
\|u(t)\|_{H^{1}} \leq C\left(u_{0}\right) e^{-\mu t}
$$

for all $t>0$, and for some constant $C\left(u_{0}\right)$ depending on $u_{0}$.

Hence, if $\psi(t) \in V \cap \mathcal{S}$ is a solution of (3.1) such that $\|\psi(0)-\eta\|_{H^{1}}$ is small enough, then for all $t$

$$
\|\psi(t)-\eta\|_{L^{2}} \leq C e^{-\mu t}
$$

for some constant $C$ and $\mu$.

Proof. As $A$ is symmetric, we calculate that

$$
\partial_{t}\langle u(t), A u(t)\rangle=-2\langle A u(t), A u(t)\rangle+2\langle R(u(t)), A u(t)\rangle .
$$

Using (2.10), and the fact that for all $u$ and $v$ in $H^{1},\langle u, A v\rangle \leq C\|u\|_{H^{1}}\|v\|_{H^{1}}$, we obtain

$$
\left|\partial_{t}\langle u(t), A u(t)\rangle\right| \leq-c\langle u(t), A u(t)\rangle+2 C\|R(u(t))\|_{H^{1}}\|u(t)\|_{H^{1}}
$$

for some positive constant $c$ and $C$. As we have by definition $\|R(u(t))\|_{H^{1}} \leq$ $\|u(t)\|_{H^{1}}^{2} \leq C\langle u(t), A u(t)\rangle$, we infer the estimate

$$
\left|\partial_{t}\langle u(t), A u(t)\rangle\right| \leq-c\langle u(t), A u(t)\rangle+C\langle u(t), A u(t)\rangle^{3 / 2} .
$$

If $\langle u(t), A u(t)\rangle \leq B^{2}$ at $t=0$, we check that by continuity argument, we have the rough estimate

$$
\langle u(t), A u(t)\rangle \leq B^{2} e^{-(c-C B) t},
$$

The result easily follows by taking $B$ small enough and using (2.9). 


\section{Convergence Result}

We consider the scheme described in the introduction: from a function $\psi_{n}$ of $L^{2}$ norm 1, close enough to $\eta$, we can write $\psi_{n}=\left(1+r\left(u_{n}\right)\right) \eta+u_{n}$ with $u_{n} \in W$. We then define $\psi_{n+1}$ by the relations (1.5) and (1.6). Note that as $\psi_{n+1}$ is of $L^{2}$ norm 1 , we can define $u_{n+1}$ by the formula $\psi_{n+1}=$ $\left(1+r\left(u_{n+1}\right) \eta+u_{n+1}\right.$ if $\psi_{n+1}$ is close enough to $\Gamma$. The map $u_{n} \mapsto u_{n+1}$ satisfies the following relation:

Lemma 4.1. Let $R \leq \frac{1}{4}$ be given. There exists $\tau_{0}$ such that for all $\tau \leq \tau_{0}$ the numerical scheme (1.5)-(1.6) is well defined from $\mathcal{S} \cap \mathcal{U}(R)$ to $\mathcal{S} \cap \mathcal{U}(2 R)$ and is equivalent to an application $u_{n} \mapsto u_{n+1}$ satisfying

$u_{n+1}=u_{n}+\tau P_{W}\left(\frac{1}{2} \Delta u_{n+1}-\lambda u_{n}+\eta^{2} u_{n+1}+2 \eta^{2} u_{n}\right)+\mathcal{O}\left(\tau\left\|u_{n}, u_{n+1}\right\|_{H^{1}}^{2}\right)$,

which is a time discretization of the equation

$$
\begin{aligned}
\partial_{t} u & =P_{W}\left(-\lambda u+\frac{1}{2} \Delta u+3 \eta^{2} u\right)+\mathcal{O}\left(\|u\|_{H^{1}}^{2}\right) \\
& =-A u+\mathcal{O}\left(\|u\|_{H^{1}}^{2}\right),
\end{aligned}
$$

corresponding to the normalized gradient flow system (3.1).

Proof. Note that with the assumption on $R$, we can assume that $\left\|u_{n}\right\|_{H^{1}} \leq$ $1 / 2$. Let us calculate $\left(r_{n}^{*}, u_{n}^{*}\right)$ such that

$$
\psi_{n}^{*}=\chi\left(r_{n}^{*}, u_{n}^{*}\right)=\left(1+r_{n}^{*}\right) \eta+u_{n}^{*},
$$

with $u_{n}^{*} \in W$, that is $\left\langle\eta, u_{n}^{*}\right\rangle=0$. As $r_{n}=r\left(u_{n}\right)=\mathcal{O}\left(\left\|u_{n}\right\|_{H^{1}}^{2}\right)$, we have $\left|\psi_{n}\right|^{2}=\eta^{2}+2 u_{n} \eta+\mathcal{O}\left(\left\|u_{n}\right\|_{H^{1}}^{2}\right)$. Let us assume that $\left|r_{n}^{*}\right| \leq 1$, we can expand the Hamiltonian term

$$
\begin{aligned}
& -\widehat{\nabla H}\left(\psi_{n}, \psi_{n}^{*}\right)=\frac{1}{2} \Delta \psi_{n}^{*}+\left|\psi_{n}\right|^{2} \psi_{n}^{*} \\
= & \left(1+r_{n}^{*}\right)\left(-\eta^{3}+\lambda \eta\right)+\frac{1}{2} \Delta u_{n}^{*}+\eta^{2} u_{n}^{*}+\left(\eta^{3}+2 u_{n} \eta^{2}\right)\left(1+r_{n}^{*}\right)+\mathcal{O}\left(\left\|u_{n}, u_{n}^{*}\right\|_{H^{1}}^{2}\right),
\end{aligned}
$$

and hence we find

$-\widehat{\nabla H}\left(\psi_{n}, \psi_{n}^{*}\right)=\lambda \eta\left(1+r_{n}^{*}\right)+\frac{1}{2} \Delta u_{n}^{*}+\eta^{2} u_{n}^{*}+2 u_{n} \eta^{2}\left(1+r_{n}^{*}\right)+\mathcal{O}\left(\left\|u_{n}, u_{n}^{*}\right\|_{H^{1}}^{2}\right)$.

By taking the projection $P_{\eta}$ of the equation (1.5), we obtain

$$
1+r_{n}^{*}=1+r_{n}+\tau\left(1+r_{n}^{*}\right) \lambda+2 \tau\left\langle u_{n}, \eta^{3}\right\rangle\left(1+r_{n}^{*}\right)+\mathcal{O}\left(\tau\left\|u_{n}, u_{n}^{*}\right\|_{H^{1}}^{2}\right) .
$$

Remark 4.2. At this stage, the same calculations for the implicit explicit of fully implicit schemes would yield a term depending on $\eta^{3}$ in (4.2) which is not in $W$ nor in $\mathbb{R} \eta$. After taking a projection, this term would not vanish, while the "constant" term $\lambda \eta\left(1+r_{n}^{*}\right)$ is here orthogonal to $W$ (which reflects the fact that the ground state is exactly integrated by the linearly implicit scheme). 
We deduce that for $\tau \leq \tau_{0}$ small enough with respect to $R$ (and $\lambda$ )

$$
\begin{aligned}
1+r_{n}^{*} & =\frac{1+r_{n}}{1-\tau \lambda-2 \tau\left\langle u_{n}, \eta^{3}\right\rangle}+\mathcal{O}\left(\tau\left\|u_{n}, u_{n}^{*}\right\|_{H^{1}}^{2}\right) \\
& =\frac{1}{1-\tau \lambda}\left(1+r_{n}+\frac{2 \tau}{1-\tau \lambda}\left\langle u_{n}, \eta^{3}\right\rangle\right)+\mathcal{O}\left(\tau\left\|u_{n}, u_{n}^{*}\right\|_{H^{1}}^{2}\right),
\end{aligned}
$$

and by taking the projection on $W$, we obtain

$$
u_{n}^{*}=u_{n}+\tau P_{W}\left(\frac{1}{2} \Delta u_{n}^{*}+\eta^{2} u_{n}^{*}+\frac{2}{1-\tau \lambda} u_{n} \eta^{2}\right)+\mathcal{O}\left(\tau\left\|u_{n}, u_{n}^{*}\right\|_{H^{1}}^{2}\right) .
$$

Let us now calculate and asymptotic expansion of the $L^{2}$ norm of $\psi_{n}^{*}$,

$$
\left\|\psi_{n}^{*}\right\|_{L^{2}}^{2}=\left(1+r_{n}^{*}\right)^{2}+\left\|u_{n}^{*}\right\|_{L^{2}}^{2} .
$$

We have

$$
\begin{aligned}
\left(1+r_{n}^{*}\right)^{2} & =\left(\frac{1}{1-\tau \lambda}\right)^{2}\left(1+r_{n}+\frac{2 \tau}{1-\tau \lambda}\left\langle u_{n}, \eta^{3}\right\rangle\right)^{2}+\mathcal{O}\left(\tau\left\|u_{n}, u_{n}^{*}\right\|_{H^{1}}^{2}\right) \\
& =\left(\frac{1}{1-\tau \lambda}\right)^{2}\left(\left(1+r_{n}\right)^{2}+\frac{4 \tau}{1-\tau \lambda}\left\langle u_{n}, \eta^{3}\right\rangle\right)+\mathcal{O}\left(\tau\left\|u_{n}, u_{n}^{*}\right\|_{H^{1}}^{2}\right)
\end{aligned}
$$

and

$$
\left\|u_{n}^{*}\right\|_{L^{2}}^{2}=\left\|u_{n}\right\|_{L^{2}}^{2}+\mathcal{O}\left(\tau\left\|u_{n}, u_{n}^{*}\right\|_{H^{1}}^{2}\right) .
$$

Hence we calculate that

$$
\begin{aligned}
& \left\|\psi_{n}^{*}\right\|_{L^{2}}^{-1}=\left(\left(1+r_{n}^{*}\right)^{2}+\left\|u_{n}^{*}\right\|_{L^{2}}^{2}\right)^{-1 / 2} \\
& =(1-\tau \lambda)\left(\left(1+r_{n}\right)^{2}+\frac{4 \tau}{1-\tau \lambda}\left\langle u_{n}, \eta^{3}\right\rangle+\left\|u_{n}\right\|_{L^{2}}^{2}+\mathcal{O}\left(\tau\left\|u_{n}, u_{n}^{*}\right\|_{H^{1}}^{2}\right)\right)^{-1 / 2} \\
& =(1-\tau \lambda)\left(1+\frac{1}{1-\tau \lambda} 4 \tau\left\langle u_{n}, \eta^{3}\right\rangle+\mathcal{O}\left(\tau\left\|u_{n}, u_{n}^{*}\right\|_{H^{1}}^{2}\right)\right)^{-1 / 2} \\
& =1-\tau \lambda-2 \tau\left\langle u_{n}, \eta^{3}\right\rangle+\mathcal{O}\left(\tau\left\|u_{n}, u_{n}^{*}\right\|_{H^{1}}^{2}\right) .
\end{aligned}
$$

Let us rewrite (4.3) as

$$
u_{n}^{*}-\tau P_{W}\left(\frac{1}{2} \Delta u_{n}^{*}+\eta^{2} u_{n}^{*}\right)=u_{n}+\tau P_{W}\left(\frac{2}{1-\tau \lambda} u_{n} \eta^{2}\right)+\mathcal{O}\left(\tau\left\|u_{n}, u_{n}^{*}\right\|_{H^{1}}^{2}\right) .
$$

Hence by multiplying by $\left\|\psi_{n}^{*}\right\|_{L^{2}}^{-1}$ we obtain

$$
\begin{aligned}
u_{n+1}-\tau P_{W}\left(\frac{1}{2} \Delta u_{n+1}\right. & \left.+\eta^{2} u_{n+1}\right) \\
= & u_{n}-\tau \lambda u_{n}+\tau P_{W}\left(2 u_{n} \eta^{2}\right)+\mathcal{O}\left(\tau\left\|u_{n}, u_{n+1}\right\|_{H^{1}}^{2}\right),
\end{aligned}
$$

which yields the result.

We can now prove the main result of this section:

Theorem 4.3. There exists constants $R, \tau_{0}, r$ and $C$ such that if $\psi_{0} \in$ $\mathcal{S} \cap \mathcal{U}(R)$, that for all $\tau \leq \tau_{0}$ the solution of the numerical scheme (1.5)(1.6) satisfies

$$
\forall n \quad\left\|\psi_{n}-\eta\right\|_{H^{1}} \leq C e^{-r n \tau} .
$$


Proof. Note that with the previous notations, we have $\left\|\psi_{n}-\eta\right\|_{H^{1}}=\mathcal{O}\left(\left\|u_{n}\right\|_{H^{1}}\right)$. We will prove the following result, which implies (4.4) after a slight change of constants: There exists $B, \tau_{0}, r$ and $C$ such that if $\left\|u_{0}\right\|_{H^{1}} \leq B$ and $\tau \leq \tau_{0}$, then every sequence $\left(u_{n}\right)_{n \geq 0}$ defined by (4.1) satisfies the estimate

$$
\forall n \quad\left\|u_{n}\right\|_{H^{1}} \leq C e^{-r n \tau} .
$$

We rewrite the system (4.1) as

$$
u_{n+1}=u_{n}-\tau\left(L u_{n+1}+B u_{n}\right)+\mathcal{O}\left(\tau\left\|u_{n}, u_{n+1}\right\|_{H^{1}}^{2}\right),
$$

with

$$
L u=P_{W}\left(\frac{1}{2} \Delta u+\eta^{2} u\right) \text { and } B u=P_{W}\left(-\lambda u+2 \eta^{2} u\right) .
$$

We know that $A=L+B$ is coercive in $H^{1}$. Note moreover that $B$ is a symmetric bounded operator in $W$. We can write

$$
u_{n+1}=u_{n}-\tau(L+B) u_{n+1}+\tau B\left(u_{n}-u_{n+1}\right)+\mathcal{O}\left(\tau\left\|u_{n}, u_{n+1}\right\|_{H^{1}}^{2}\right) .
$$

Now for $\tau$ sufficiently small, the operator $I+\tau B$ is symmetric, invertible, positive and bounded from $W$ to itself. Hence we can rewrite the numerical scheme as

$$
u_{n+1}=u_{n}-\tau(I+\tau B)^{-1}(L+B) u_{n+1}+\mathcal{O}\left(\tau\left\|u_{n}, u_{n+1}\right\|_{H^{1}}^{2}\right) .
$$

Setting $v_{n}=(I+\tau B)^{1 / 2} u_{n}$, we obtain the relation

$$
v_{n+1}=v_{n}-\tau(I+\tau B)^{-1 / 2}(L+B)(I+\tau B)^{-1 / 2} v_{n+1}+\mathcal{O}\left(\tau\left\|v_{n}, v_{n}^{*}\right\|_{H^{1}}^{2}\right) .
$$

In this new variable $v_{n}$, we thus can write the induction relation

$$
v_{n+1}=v_{n}-\tau A_{\tau} v_{n+1}+\tau w_{n}
$$

with $A_{\tau}=(I+\tau B)^{-1 / 2}(L+B)(I+\tau B)^{-1 / 2}$,

$$
w_{n}=\mathcal{O}\left(\left\|v_{n}, v_{n+1}\right\|_{H^{1}}^{2}\right) \quad \text { and } \quad c\|v\|_{H^{1}}^{2} \leq\left\langle A_{\tau} v, v\right\rangle_{L^{2}} \leq C\|v\|_{H^{1}}^{2},
$$

for some constants $c$ and $C$ independent of $\tau$. Note also that the inequality (2.10) holds for the operators $A_{\tau}$ uniformly in $\tau \leq \tau_{0}$ sufficiently small. From (4.5), we can write

$$
\begin{array}{r}
\left\langle A_{\tau} v_{n}+\tau A_{\tau} w_{n}, v_{n}+\tau w_{n}\right\rangle_{L^{2}}=\left\langle A_{\tau} v_{n+1}+\tau A_{\tau}^{2} v_{n+1}, v_{n+1}+\tau A_{\tau} v_{n+1}\right\rangle_{L^{2}} \\
=\left\langle A_{\tau} v_{n+1}, v_{n+1}\right\rangle+2 \tau\left\langle A_{\tau} v_{n+1}, A_{\tau} v_{n+1}\right\rangle_{L^{2}}+\tau^{2}\left\langle A_{\tau}^{2} v_{n+1}, A_{\tau} v_{n+1}\right\rangle_{L^{2}} \\
\geq(1+2 c \tau)\left\langle A_{\tau} v_{n+1}, v_{n+1}\right\rangle
\end{array}
$$

using (2.10) and the fact that $\left\langle A_{\tau}^{2} v_{n+1}, A_{\tau} v_{n+1}\right\rangle_{L^{2}} \geq 0$.

On the other hand we have

$$
\left\langle A_{\tau} v_{n}+\tau A_{\tau} w_{n}, v_{n}+\tau w_{n}\right\rangle_{L^{2}}=\left\langle A_{\tau} v_{n}, v_{n}\right\rangle+2 \tau\left\langle A_{\tau} v_{n}, w_{n}\right\rangle+\tau^{2}\left\langle A_{\tau} w_{n}, w_{n}\right\rangle .
$$

But using an integration by part in the unbounded part of $A_{\tau}$, we have for all $\tau \leq \tau_{0}$ sufficiently small, and for any $v, w$ in $H^{1}$

$$
\left|\left\langle A_{\tau} v, w\right\rangle\right| \leq C\|v\|_{H^{1}}\|w\|_{H^{1}} .
$$

Using the estimate on $w_{n}$, we thus see that if $\left\langle A_{\tau} v_{n}, v_{n}\right\rangle$ and $\left\langle A_{\tau} v_{n+1}, v_{n+1}\right\rangle$ are bounded by $B^{2}$, we have

$$
\left\langle A_{\tau} v_{n}+\tau A_{\tau} w_{n}, v_{n}+\tau w_{n}\right\rangle_{L^{2}} \leq(1+C \tau B)\left\langle A_{\tau} v_{n}, v_{n}\right\rangle+C \tau B\left\langle A_{\tau} v_{n+1}, v_{n+1}\right\rangle .
$$


The previous inequalities imply:

$$
\left\langle A_{\tau} v_{n+1}, v_{n+1}\right\rangle_{L^{2}} \leq \frac{1+C B \tau}{1+c \tau-C B \tau}\left\langle A_{\tau} v_{n}, v_{n}\right\rangle_{L^{2}}
$$

as long as $\left\langle A_{\tau} v_{n}, v_{n}\right\rangle \leq B^{2}$ and $\left\langle A_{\tau} v_{n+1}, v_{n+1}\right\rangle \leq B^{2}$. If $B$ is small enough (independently of $\tau$ ) we have

$$
\frac{1+C B \tau}{1+c \tau-C B \tau} \leq e^{-a \tau}
$$

for some constant $a$ independent of $\tau$. This implies that

$$
\left\langle A_{\tau} v_{n}, v_{n}\right\rangle_{L^{2}} \leq B^{2} e^{-a n \tau},
$$

for all $n$, by an induction argument. This shows the result.

Remark 4.4. If we choose another discretization - implicit explicit or fully implicit, see (1.7) - then as explained in Remark 4.2 the term in $\eta^{3}$ do not disapear in the equation, and after some manipulations we end up with a recursion formula of the form

$$
v_{n+1}=v_{n}+\tau^{2} g-\tau A_{\tau} v_{n+1}+\tau \rho_{n}
$$

with $\rho_{n}=\mathcal{O}\left(\left\|v_{n}, v_{n+1}\right\|_{H^{1}}^{2}\right)$ and $g \in W$ a non zero function. It is relatively easy to prove that in this case, $v_{n}$ converges towards a function $v_{\tau}^{\infty}=\tau A_{\tau}^{-1} g+\mathcal{O}\left(\tau^{2}\right)$. Indeed, the previous equation if a discretization of the modified problem

$$
\partial_{t} v=\tau g-A_{\tau} v+B(v)
$$

whose solution exponentially converges towards the solution of the problem

$$
\tau g-A_{\tau} v+B(v)=0
$$

which exists by standard implicit function theorem in $H^{1}$. This shows that in this case, $\psi_{n}$ converges exponentially in $H^{1}$ towards a modified soliton $\eta_{\tau}=\eta+\mathcal{O}(\tau)$. As the result is weaker than for the linearly implicit scheme, we do not give more mathematical details.

\section{Fully discrete CASE}

The goal of this last section is to show that the previous results carry over to fully discrete systems. This fact relies essentially on the results in [3] and [6]: After discretization of the previous system by finite difference method, there exists a discrete ground state $\eta_{h}$ minimizing a discrete convex functional $\mathcal{H}_{h}$ which is an approximation of the exact functional $\mathcal{H}$ defined above. Here $h$ denotes the space discretization parameter and $\eta_{h}$ is close to $\eta$ with an error of order $h$. Moreover, the same holds true after Dirichlet cut-off upon adding an exponentially decreasing error term, and it can be proven that the discrete functionals satisfy the same estimates as the continuous ones, uniformly with respect to the discretization parameters.

Having fixed a positive parameter $h$ we discretize in space by substituting the sequence $\psi^{\ell} \simeq \psi(h \ell), \ell \in \mathbb{Z}$ for the function $\psi(x)$, and the second order operator of finite difference $\Delta_{h}$ defined by

$$
\left(\Delta_{h} \psi\right)^{\ell}:=\frac{\psi^{\ell+1}+\psi^{\ell-1}-2 \psi^{\ell}}{h^{2}},
$$


for the Laplace operator $\partial_{x x}$. We also impose Dirichlet boudary conditions for $|j| \geq K+1$, and the parabolic equation (1.4) then becomes

$$
\left\{\begin{aligned}
\frac{\mathrm{d}}{\mathrm{d} t} \psi^{\ell} & =\frac{1}{2 h^{2}}\left(\psi^{\ell+1}+\psi^{\ell-1}-2 \psi^{\ell}\right)+\left|\psi^{\ell}\right|^{2} \psi^{\ell}, \quad \ell \in \mathbb{Z}, \quad|\ell| \leq K, \\
\psi^{\ell} & =0, \quad|\ell| \geq K+1,
\end{aligned}\right.
$$

where $t \mapsto \psi(t)=\left(\psi^{\ell}(t)\right)_{\ell \in \mathbb{Z}}$ is an application from $\mathbb{R}$ to $\mathbb{R}^{\mathbb{Z}}$. With this equation is associated a Hamiltonian function and a discrete $L^{2}$ norm given by

$$
H_{h}(\psi)=h \sum_{j \in \mathbb{Z}}\left[\left|\frac{\psi^{j}-\psi^{j-1}}{h}\right|^{2}-\frac{\left|\psi^{j}\right|^{4}}{2}\right] \quad \text { and } \quad N_{h}(\psi)=h \sum_{j \in \mathbb{Z}}\left|\psi^{j}\right|^{2}
$$

The discrete space of functions is

$$
V_{h, K}=\left\{\psi^{j} \in \mathbb{C}^{\mathbb{Z}} \mid \psi^{j}=\psi^{-j}, \quad \psi^{j}=0 \quad \text { for } \quad j>K\right\}
$$

equipped with the discrete $H^{1}$ norm

$$
\|\psi\|_{h}^{2}=2 h \sum_{j \in \mathbb{Z}} \frac{\left|\psi^{j+1}-\psi^{j}\right|^{2}}{h^{2}}+h \sum_{j \in \mathbb{Z}}\left|\psi^{j}\right|^{2},
$$

and we also define the scalar product

$$
\langle\psi, \varphi\rangle_{h}:=h \sum_{j \in \mathbb{Z}} \psi^{j} \varphi^{j}
$$

Following [6, 3], we identify $V_{h}$ with a finite element subspace of $H^{1}(\mathbb{R})$. More precisely, defining the function $s: \mathbb{R} \rightarrow \mathbb{R}$ by

$$
s(x)= \begin{cases}0 & \text { if } \quad|x|>1 \\ x+1 & \text { if } \quad-1 \leq x \leq 0 \\ -x+1 & \text { if } \quad 0 \leq x \leq 1\end{cases}
$$

the identification is done through the map $i_{h}: V_{h} \rightarrow H^{1}(\mathbb{R})$ defined by

$$
\left\{\psi_{j}\right\}_{j \in \mathbb{Z}} \mapsto\left(i_{h} \psi\right)(x):=\sum_{j \in \mathbb{Z}} \psi_{j} s\left(\frac{x}{h}-j\right) .
$$

The main results in [6] and [3] can expressed as follows:

Theorem 5.1. For h sufficiently small and $K$ sufficiently large, there exists a unique real minimizer $\eta_{h, K}=\left(\eta_{h, k}^{j}\right)_{j \in \mathbb{Z}} \in V_{h, K}$ of the functional $H_{h}(\psi)$ over the set

$$
\left\{\psi=\left(\psi^{j}\right)_{j \in \mathbb{Z}} \in V_{h, K}, \quad N_{h}(\psi)=1\right\},
$$

equipped with the norm (5.4). Moreover, $\eta_{h, K}$ satisfies the equation

$$
\begin{aligned}
\frac{1}{2}\left(\Delta_{h} \eta_{h, K}\right)^{\ell}+\left|\eta_{h, K}^{\ell}\right|^{2} \eta_{h, K}^{\ell} & =\lambda_{h} \eta_{h, K}^{\ell}, \quad \ell=-K, \ldots, K, \\
\eta_{h, K}^{\ell} & =0, \quad|\ell| \geq K+1,
\end{aligned}
$$

for some $\lambda_{h}>0$. Moreover, we can define a local coordinate system $\psi=$ $\chi_{h}(r, u)=(1+r) \eta_{h, K}+u$ in a vicinity of $\eta_{h, K}$ in $V_{h, K}$ with $u \in W_{h}:=\{u \in$ $\left.V_{h, K}, \quad\left\langle u, \eta_{h, K}\right\rangle_{h}=0\right\}$, and such that if $r_{h}(u)$ is defined by the implicit relation $\left.N_{h}\left(\chi_{h}\left(r_{h}(u), u\right)\right)\right)=1$, then the functional $\left.\mathcal{H}_{h}(u)=H_{h}\left(\chi_{h}\left(r_{h}(u), u\right)\right)\right)$ 
has a unique non degenerate minimum in $u=0$ in $V_{h, K}$ and satisfies a convexity estimate of the form (2.7) uniformly in $h$ and $K$.

Finally, the discrete ground state $\eta_{h, K}$ is close to the continuous one in the sense that

$$
\left\|i_{h} \eta_{h, K}-\eta\right\|_{H^{1}} \leq C\left(h+\frac{1}{h^{2}} e^{-C_{1} h K}\right),
$$

where $C$ and $C_{1}$ do not depend on $h$, and where $i_{h}$ is the embedding (5.6).

The fully discrete algorithm corresponding to (1.5)-(1.6) then consists in the two following steps: From $\left(\psi_{n}^{\ell}\right)_{j=-K}^{K}$ such that $N_{h}\left(\psi_{n}\right)=1$,

(i) Compute $\psi_{n}^{*}=\left(\psi_{n}^{*, \ell}\right)_{j \in \mathbb{Z}}$ defined by the relation

$$
\left\{\begin{array}{l}
\psi_{n}^{*, \ell}=\psi_{n}^{\ell}+\tau\left(\frac{1}{2}\left(\Delta_{h} \psi_{n}^{*}\right)^{\ell}+\left|\psi_{n}^{\ell}\right|^{2} \psi_{n}^{*, \ell}\right), \quad \ell \in \mathbb{Z}, \quad|\ell| \leq K, \\
\psi^{*, \ell}=0, \quad|\ell| \geq K+1 .
\end{array}\right.
$$

(ii) Normalization step:

$$
\psi_{n+1}^{\ell}=\frac{\psi_{n}^{*, \ell}}{N_{h}\left(\psi_{n}^{*}\right)} .
$$

The equation (5.7) guarantees that this fully discrete algorithm preserves exactly the discrete ground state $\eta_{h, K}$. We can now copy the proof of the continuous case an adapt it directly to the fully discrete case: We can prove that

$$
\left\|\psi_{n}-\eta_{h, K}\right\|_{h} \leq C e^{-c n \tau}, \quad n \geq 0,
$$

for some constants $C$ and $c$ independent of $h, K$ and $\tau$. By using the previous estimate, we obtain the following result:

Theorem 5.2. There exist constants $B, C, C_{1}$ and $c$ such that for $h, \tau$ sufficiently small and $K$ sufficiently large, if $\left(\psi_{0}\right)=\left(\psi_{0}^{\ell}\right)_{\ell=-K}^{K}$ satisfies

$$
\left\|i_{h} \psi_{0}-\eta\right\|_{H^{1}} \leq B
$$

then the solution $\psi_{n}$ of the fully discrete algorithm (5.9)-(5.10) satisfies

$$
\left\|i_{h} \psi_{n}-\eta\right\|_{H^{1}} \leq C\left(e^{-c n \tau}+h+\frac{1}{h^{2}} e^{-C_{1} h K}\right) .
$$

Remark 5.3. Following Remark 4.4, we can prove a similar result for the implicit-explicit and fully implicit algorithms (1.7), but the fully discrete schemes will converge towards a discrete ground state $\eta_{\tau, h, K}$ satisfying

$$
\left\|i_{h} \eta_{\tau, h, K}-\eta\right\|_{H^{1}} \leq C\left(\tau+h+\frac{1}{h^{2}} e^{-C_{1} h K}\right),
$$

and the previous result has to be modified accordingly in these cases.

\section{REFERENCES}

[1] S.K. Adhikari, Numerical solution of the two-dimensional Gross-Pitaevskii equation for trapped interacting atoms, Phys. Lett. A, 265 (2000) 91-96.

[2] S.K. Adhikari, Numerical solution of spherically symmetric Gross-Pitaevskii equation in two space dimensions, Phys. Lett. E, 62 (2000) 2937-2944.

[3] D. Bambusi and T. Penati Continuous approximation of breathers in one and two dimensional DNLS lattices, Nonlinearity 23 (2010), no. 1, 143157. 
[4] W. Bao and Q. Du, Computing the ground state solution of Bose-Einstein condensates by a normalized gradient flow. SIAM J. Sci. Comput. 25 (2004) 1674-1697.

[5] W. Bao and W. Tang, Ground state solution of trapped interacting Bose-Einstein condensate by directly minimizing the energy functional, J. Comput. Phys. 187 (2003), $230-254$.

[6] D. Bambusi, E. Faou and B. Grébert Existence and stability of ground states for fully discrete approximations of the nonlinear Schrödinger equation. Numer. Math. 123 (2013) 461-492

[7] M.L. Chiofalo, S. Succi and M.P. Tosi, Ground state of trapped interacting BoseEinstein condensates by an explicit imaginary time algorithm, Phys. Rev. E, 62 (2000) $7438-7444$.

[8] M. Edwards and K. Burnett, Numerical solution of the nonlinear Schrödibnger equation for small samples of trapped neutral atoms, Phys. Rev. 1, 51 (1995) 1382-1386.

[9] J. Froehlich, S. Gustafson, L. Jonsson and I.M. Sigal Solitary wave dynamics in an external potential. Comm. Math. Phys. 250 (2004), 613-642.

[10] M. Grillakis, H. Shatah and W. Strauss, Stability theory of solitary waves in the presence of symmetry. I., J. Funct. Anal. 74 (1987) 160-197.

[11] M. Grillakis, H. Shatah and W. Strauss, Stability theory of solitary waves in the presence of symmetry. II., J. Funct. Anal. 94 (1990) 308-348.

[12] E. Hairer and Christian Lubich, Energy-diminishing integration of gradient systems, IMA J. Numer. Anal. 34 (2014), 452-461.

[13] M. I. Weinstein, Modulational stability of ground states of nonlinear Schrödinger equations, SIAM J. Math. Anal. 16 (1985) 472-491.

INRIA \& IRMAR. ENS Rennes, Avenue Robert Schumann F-35170 Bruz, FRANCE

E-mail address: Erwan.Faou@inria.fr

iUt de Lannion, Université de Rennes 1. Rue Edouard Branly, BP 30219, 22302 Lannion Cedex, France.

E-mail address: Tiphaine. Jezequel@univ-rennes1.fr 